\title{
LA CONFIANZA Y EL CUIDADO EN EL LIDERAZGO ESCOLAR DE DIRECTORAS CHILENAS
}

\author{
Andrea Carrasco ${ }^{1}$, Diego Barraza ${ }^{2}$
}

\begin{abstract}
RESUMEN
Las discusiones actuales respecto del liderazgo escolar han hecho hincapié en el valor de las relaciones interpersonales al interior de la escuela, a la hora de potenciar la mejora de las comunidades educativas. A su vez, han señalado que estas juegan un rol preponderante en contextos de vulnerabilidad. En este marco, el presente artículo busca reflexionar acerca de cómo se expresan las prácticas de confianza y cuidado en los discursos referentes al ejercicio de liderazgo de directoras de establecimientos educativos de la Región Metropolitana. Esta investigación fue de carácter cualitativo y contó con los discursos de diez directoras. Las reflexiones aquí presentadas se enmarcan en un proceso investigativo más amplio, que conllevó una investigación posicionada desde un enfoque biográfico narrativo. Entre los principales resultados destaca la existencia de una preocupación permanente, de parte de las directoras, por desarrollar prácticas que fomenten el cuidado al interior de los equipos de trabajo y la construcción de un clima de confianza en el marco de las relaciones que se establecen en las comunidades educativas.
\end{abstract}

Conceptos clave: confianza, cuidado, mujeres, directoras escolares.

\section{TRUST AND CARE PRACTICES OF FEMALE CHILEAN PRINCIPALS AND THEIR LEADERSHIP IN THE SCHOOL}

\begin{abstract}
Current discussions regarding school leadership have emphasized the value of interpersonal relationships within the school to enhance the improvement of educational communities. In turn, they have pointed out that this plays a preponderant role in contexts of vulnerability. Within this framework, this article seeks to reflect on how practices of trust and care are expressed in the discourses concerning the exercise of leadership by female principals of educational establishments in the metropolitan region. This research was of a qualitative nature and included the discourse of ten female principals. The reflections presented here are part of a broader research process, which involved research based on a biographical and narrative approach. Among the main results, the existence of a permanent concern on the part of the principals to develop practices that foster care within work teams and the construction of a climate of trust within the framework of the relationships established in the educational communities.
\end{abstract}

Key concepts: trust, care, women, school principal.

Universidad de Chile, Santiago, Chile. Contacto: ancarrasco@uchile.cl

2 Universidad de Chile, Santiago, Chile. Contacto: diego.barraza.r@ug.uchile.cl 


\section{Introducción}

Diversas investigaciones sugieren que la calidad de los directivos y sus capacidades de liderazgo son un factor relevante a la hora de pensar la posibilidad de mejora de las comunidades escolares (MINEDUC, 2015). Por tal razón, no resulta extraño que las políticas educativas piensen en los directivos como actores claves al momento de proyectar el desarrollo y/o fortalecimiento de los aprendizajes en las y los estudiantes (Campos, Valdés y Ascorra, 2019). A este respecto, la literatura revela la existencia de una correlación entre el buen desempeño de un liderazgo directivo y la mejora en los resultados de aprendizajes en los establecimientos educacionales (Bolívar, 2010; Horn y Marfán, 2010; Guimaraes y Valenzuela, 2016).

Si bien gran parte del efecto provocado por las y los directivos en esta materia depende de agentes que median en su relación con los estudiantes — los docentes- (Waters Marzano \& McNulty, 2003; Leithwood, Louis, Anderson \& Wahlstrom, 2004; Robinson, 2007; Leithwood y Jantzi, 2008), su injerencia no deja de ser relevante, cuestión que es posible evidenciar si focalizamos la mirada en el caso chileno (Weinstein y Muñoz, 2012, Vanni et al., 2018).

En este marco, se ha destacado el último tiempo que el desarrollo cognitivo y socioemocional de los estudiantes depende no solo de elementos técnicos, sino de una serie de factores que se encuentran, se relacionan e interactúan al interior de las comunidades escolares (Louis \& Murphy, 2019). Por esto, el desafío de la mejora en el rendimiento escolar requiere de la construcción de mejores entornos de aprendizaje, tanto para docentes como para estudiantes, así como de la creación de condiciones escolares facilitadoras (Lee $\&$ Louis, 2019).

A raíz de esto, al pensar cómo potenciar la mejora en las escuelas, cada vez se ha otorgado mayor valor a las relaciones interpersonales, destacando en ellas elementos como la confianza (Brezicha, Bergmark \& Mitra, 2015) o el cuidado (Louis \& Murphy, 2017), componentes considerados muy relevantes en la actualidad para pensar el desarrollo de las comunidades educativas, sobre todo en contextos vulnerables (Louis \& Murphy, 2019). 
Así, durante el último tiempo han surgido una serie de líneas paralelas de investigación que han buscado ahondar en la injerencia de estos componentes en las prácticas de liderazgo y su relación con la mejora de las culturas escolares. Entre estas encontramos el liderazgo positivo (Ibíd.), el liderazgo inclusivo (Ryan, 2016) y el liderazgo centrado en el cuidado — caring leadership - (Smylie, Murphy \& Louis, 2016). Estos liderazgos otorgan gran importancia a las relaciones que se viven en las escuelas, en la medida en que ellas permiten la construcción de entornos que facilitan el aprendizaje (Louis \& Murphy, 2018). Entre estos enfoques destacan dos conceptos que presentan gran relevancia para el presente artículo: el cuidado (Lomotey y Lowery, 2015; Louis, Murphy \& Smylie, 2016; Louis \& Murphy, 2017; Schechter y Shaked, 2017) y la confianza (Torre \& Murphy, 2014; Louis \& Murphy, 2017).

En relación con lo anterior, en Chile pareciera existir un creciente interés por trabajar en las relaciones que se establecen desde el liderazgo. En este contexto, la encuesta TALIS (OCDE, 2018) señala la existencia de espacios que motivan la colaboración y la participación al interior de la escuela. A este respecto, según los datos, el $71 \%$ de los docentes reconocen trabajar en una cultura escolar colaborativa, caracterizada por el apoyo mutuo, cifra alta que, aun cuando no se equipara al promedio de los países de la OCDE (81\%), nos habla de una tendencia que avanza hacia ese horizonte.

Según los mismos datos, el 79\% de los directores en Chile tomaron medidas específicas para apoyar la colaboración entre los profesores y desarrollar nuevas prácticas docentes en los 12 meses anteriores a la encuesta, cuestión que refuerza este interés latente por construir relaciones sólidas como base de los procesos de aprendizaje.

Pero estos datos no representan un caso aislado, sino que más bien se enmarcan en un esfuerzo, por parte de la política educativa durante la última década, por posicionar estas temáticas como relevantes en lo que refiere al ejercicio del liderazgo escolar. A este respecto, se destaca una preocupación por las herramientas socioemocionales e intrapersonales en dos documentos que guían el quehacer de los directivos, a saber: El Marco para la Buena Dirección y 
el Liderazgo Escolar (2015) y Estándares Indicativos de Desempeño para los Establecimientos Educacionales y sus Sostenedores (2014).

Otra tendencia que se ha dado en el país es el aumento sostenido, en las últimas décadas, de directoras en establecimientos educativos (MINEDUC, 2019), cuestión que resulta de gran interés por aquellas tesis que señalan que el liderazgo de directoras tiende a estar más focalizado en el cuidado, la confianza y otras características interpersonales (Blackmore, 2006; Coleman, 2007; Tronto, 2018), debido a la condición histórica que se atribuye a las mujeres de "cuidadoras del hogar" (Gilligan, 2013).

El presente artículo tiene por objetivo conocer y reflexionar acerca de cómo los discursos sobre las prácticas de liderazgo de directoras de establecimientos educativos de la Región Metropolitana expresan las cualidades de confianza y cuidado ${ }^{3}$. En este sentido, se asume que el estudio es de carácter exploratorio y focalizado en las voces de las directoras entrevistadas. Por tanto, de aquí en más, el desafío está en estudiar los impactos de estas prácticas declaradas desde una perspectiva relacional y multiactoral, que permita comprender en profundidad los efectos en las comunidades educativas.

\section{Marco conceptual}

\section{a) Liderazgo escolar}

Existe cierto consenso en posicionar al liderazgo escolar como un elemento de gran influencia a la hora de pensar la posibilidad de mejora en las instituciones educativas (Leithwood, 2009; Robinson, Hopega \& Lloyd, 2009; Bolívar, 2019). En este marco, la influencia de los directores solo se ve superada por la de los docentes a la hora de pensar en las posibilidades de mejora de los aprendizajes de los estudiantes (Leithwood, Louis, Anderson y Wahlstrom, 2004; Louis, Leithwood, Wahlstrom y Anderson, 2010).

Este artículo es parte de una investigación más extensa, en la que se aborda la construcción de conocimiento profesional de directoras escolares y las características del liderazgo femenino. 
Por otra parte, es posible señalar que las prácticas de liderazgo escolar resultan ser un pilar fundamental para entender el desempeño de las comunidades de aprendizaje, sobre todo cuando se pone como parámetro de evaluación el éxito escolar. A este respecto, las investigaciones confluyen en que, por un lado, el liderazgo es de gran importancia para éste (Harris y Chapman, 2002), mientras que, por otro, un liderazgo débil es una de las principales causas de los declives que presentan las escuelas (Leithwood, Harris y Strauss, 2010).

Pero las prácticas de liderazgo nunca son un elemento estático, sino que se ven mediadas por el contexto en el que se construyen y desarrollan sus comunidades. Por esto, como señalan Leithwood, Harris y Strauss (2010), las circunstancias contextuales, compositivas, cíclicas, contingentes y condicionales pueden ser factores que dificulten el rol del liderazgo en el marco del desarrollo de las instituciones educativas.

De esta forma, es indudable que las prácticas de liderazgo de cada directora se constituyen como un factor relevante en el marco de la posibilidad de alcanzar las metas estipuladas para cada comunidad educativa, ya que son las encargadas de potenciar y movilizar a los actores, pudiendo conducir el modelo de aprendizaje de cada escuela hacia aquellos que se presenten como los más integrales para los estudiantes (McKinsey \& Company, 2008; Leithwood, 2009; Bolívar, 2012).

A raíz de esto, la literatura internacional ha tendido a otorgar cada vez mayor valor a elementos presentes en las relaciones interpersonales al interior de la escuela para potenciar la mejora de las comunidades educativas. En este marco, elementos como la confianza (Brezicha, Bergmark \& Mitra, 2015) o el cuidado (Louis $\&$ Murphy, 2017) se presentan en la actualidad como componentes fundamentales para pensar el desarrollo de las escuelas y el liderazgo, sobre todo en contextos vulnerables (Louis \& Murphy, 2019). Por lo anterior, se profundizará en los enfoques de liderazgo que se centran en los temas de confianza y cuidado. 


\section{b) Liderazgo positivo}

El liderazgo positivo deriva del enfoque de la psicología positiva, que durante mucho tiempo se ha utilizado en los estudios organizacionales y que ha tenido una presencia creciente en las discusiones educativas (Seligman, Ernst, Gillham, Reivich \& Linkins, 2009). Para este enfoque, lo importante en el marco de una organización es desarrollar prácticas de liderazgo que permitan construir entornos que potencien el desarrollo de las personas. Así, las organizaciones positivas son aquellas en las cuales los individuos están motivados porque ven que su trabajo es significativo (Louis \& Murphy, 2018), así como también son lugares que refuerzan los sentidos de sí mismo y la construcción de una identidad organizacional (Dutton, Roberts \& Bednar, 2010).

El liderazgo escolar positivo viene de pensar las escuelas como organizaciones en las cuales gran parte de los elementos que confluyen en la mejora de los estándares escolares son el resultado de la relación entre diversos actores presentes en esas comunidades, incluso cuando estos no son los estudiantes mismos (Youngblade et al., 2007; Riley, 2013). Desde este enfoque, cierto precedente señala que las relaciones de adultos basadas en activos son una condición previa para crear el bienestar de los estudiantes, y que solo donde todos o la mayoría de los miembros de la escuela están prosperando se puede vivenciar un aporte a la sociedad (Louis \& Murphy, 2017).

El liderazgo positivo tiene su énfasis en las relaciones y se debe procurar generar una aproximación positiva entre todos los integrantes del equipo escolar. Todo esto basándose en activos que implican prestar atención a los fundamentos morales y construir un vínculo con base valórica en las organizaciones escolares (Louis \& Murphy, 2019).

El liderazgo positivo es un acto relacional (Ibíd.), por ende, no es una característica excluyente de los directivos en el marco de las instituciones escolares, sino que docentes y estudiantes también confieren prácticas de liderazgo (Burrello, Beitz y Mann, 2016). En este sentido, el rol de los líderes escolares positivos se debe enfocar en el desarrollo de prácticas que promuevan la confianza de los colaboradores, cultivar un sentido de justicia organizacional 
y contribuir en la promoción del bien colectivo (Louis \& Murphy, 2019).

Los efectos de este liderazgo positivo no son directos, sino que ocurren porque cambian el clima y la cultura de la escuela (Louis \& Murphy, 2018). Así, para tener un impacto, los líderes deben establecer relaciones caracterizadas por valores, confianzas y consistencia en el carácter (Ibíd.). Todo esto complementado por intercambios asociados a la gestión directiva, orientados a mantener relaciones, asegurar la motivación en el trabajo y el reconocimiento de otros (De Jong \& Den Hartog, 2007).

\section{Confianza}

La generación de confianza, que configura a un liderazgo positivo desde un punto de vista interpersonal, puede ser entendida como la expectativa favorable que se tiene, individualmente o como comunidad, respecto al accionar de un tercero o de los miembros de una institución (Fukuyama, 1995).

En lo referente a la educación, muchos estudios han encontrado una correlación directa entre los niveles de confianza al interior de la comunidad educativa y el mejoramiento escolar (Bryk y Schneider, 2002; Conejeros, Rojas y Segure, 2010; Umekubo, Chrispeels y Daly, 2013; Cerna, 2014; Tschannen-Moran y Gareis, 2015; Peña, Weinstein y Raczynski, 2018; Aziz dos Santos, 2019). Por ende, no resulta extraño que, al momento de hablar sobre liderazgo escolar, la confianza surja como un elemento de gran importancia (Louis $\&$ Murphy, 2017). A este respecto, mucho se ha estudiado sobre la confianza entre docentes (Torre \& Murphy, 2014) o desde los docentes hacia los directivos, y muy poco de estos últimos para con los educadores (Smylie, Mayrowetz, Murphy, \& Louis 2007; Wahlstrom y Louis, 2008; Tschannen-Moran, 2009).

La confianza es un fenómeno multidimensional, con base en la reciprocidad (Louis \& Murphy, 2017). De esta forma, si los lideres no demuestran confianza en las competencias profesionales de los docentes, es altamente probable que los maestros tengan una visión negativa de su labor y de sus líderes (Brower, Schoorman \& Hoon, 
2000). Como señala Tschannen-Moran (2009), la confianza entre el docente y el directivo está relacionada y se influye constantemente al interior de las comunidades educativas.

En lo que respecta a la multidimensionalidad, la confianza no se distribuye de manera única, sino que se comparte entre cada uno de los actores que se encuentran en el contexto educativo (Fairholm $\&$ Fairholm, 2000). Y esta confianza no solo tiene una naturaleza, sino que suele dividirse entre aquella que repara en las capacidades profesionales y otra que tiene su foco en el aspecto afectivo. Esta última se encuentra muy relacionada al cuidado al interior de las escuelas (Louis \& Murphy, 2017).

\section{c) Liderazgo inclusivo}

Un ejemplo de liderazgo fundado en la confianza es el liderazgo de tipo inclusivo que, en su definición, propone relaciones horizontales entre los integrantes de la comunidad, en los roles y funciones que asumen los individuos y los grupos, y promueve las relaciones sociales e interacciones pedagógicas que eviten la jerarquización, razón por la cual se propone precisamente "eliminar las relaciones asimétricas de poder” (Ryan, 2016, p. 182). Con estas prácticas se busca contribuir a la inclusión como un proceso basado en la equidad, la participación y el compromiso colectivo en la construcción de una "cultura inclusiva" (León, 2012; Ryan, 2016).

Este tipo de liderazgo se distancia de otros enfoques tradicionales, que comprenden el liderazgo como una tarea exclusiva (y excluyente) de quienes ocupan ciertos cargos en la institución escolar. En situaciones ideales de liderazgo inclusivo, todos los individuos y grupos participan activamente en el diseño de políticas, toma de decisiones y otros procesos que implican influencia o poder. En este sentido, la concentración de poder es una práctica excluyente en sí misma (Ryan, 2016).

El liderazgo inclusivo apela a la forma o estilo de liderazgo y al fondo de éste. Si lo que se busca es lograr la inclusión en las escuelas y sus comunidades, la gestión ha de ser consistente con los ideales de la inclusión, para que los actores de las propias comunidades lo 
perciban y lo practiquen como un proceso basado en la equidad, la participación y el compromiso colectivo, que también está organizado para promover inclusión (Ibíd.).

\section{d) Caring leadership: un liderazgo cuidadoso}

El caring leadership surge como un enfoque que busca promover prácticas de liderazgo centradas en el "cuidado", como un elemento clave para la mejora educativa y la transformación pedagógica, permitiendo poner el ejercicio del liderazgo dentro de un rango más amplio de posibilidades de acción (Smylie, Murphy \& Louis, 2016).

\section{El cuidado}

Se puede comprender desde dos dimensiones, "la subjetiva — como preocupación, responsabilidad, disposición (care about)—, y la material —el cuidado como acción, ocupación (care for)—" (Molinier \& Lagarreta, 2016, p. 1).

Por otra parte, el cuidado, según lo entiende Noddings, es una condición natural, ya que "como seres humanos queremos cuidar y ser cuidados. El cuidado es importante en sí mismo" (1984, p. 7). En ese sentido, es una condición anhelada que motiva el desarrollo de esfuerzos, en la medida en que permite una valoración moral. Según Smylie, Murphy y Louis (2016), el cuidado siempre está más allá que la acción misma de cuidar, porque implica comprender cómo se hace y por qué. Noddings señala que el cuidado debe considerarse desde una perspectiva relacional, en la que tanto el cuidador como el cuidado contribuyen significativamente a la relación. "El cuidador atiende — escucha las necesidades expresadas por el cuidadoy responde de una manera que satisface la necesidad o explica satisfactoriamente por qué no se puede satisfacer la necesidad" (Noddings, 2001, p. 36).

El cuidado históricamente ha estado vinculado a "la moralidad de las mujeres" (Gilligan, 2013), pero no porque sea una situación adquirida naturalmente, sino porque ha sido una construcción social de género en la que, a las mujeres, desde pequeñas, se les atribuye este rol. Es una característica esencialmente femenina, ya 
que surge de su experiencia, lo que no quiere decir que no pueda ser compartida por los hombres (Noddings, 1984). Ahora bien, Tronto (2018) plantea que, más que asociar la ética del cuidado con la moral femenina, es necesario vincularla con una moral social, sin género, en la que los valores de la democracia se hagan carne: "el cuidado debiese ser más democrático; el proceso de cuidado debe entenderse de manera pluralista. Comprender las actividades del cuidado como aquello realmente significativo en las vidas humanas, en lugar de la economía de la producción" (Id., p. 34).

Por lo anterior, se comprende el cuidado como una función social que históricamente ha sido asociada a las mujeres, pero no por ello es un rol o tarea exclusiva de ellas. Desde aquí se asume que el cuidado cumple un rol fundamental en las metas de las instituciones educativas, ya que "puede apuntar a promover ciertos beneficios experimentales: beneficios sociales, psicológicos y emocionales que se obtienen al estar en relaciones de cuidado y sentirse atendido. El cuidado en sí mismo puede ser educativo" (Smylie, Murphy \& Louis, 2016, p. 7).

El cuidado representa una práctica compleja que requiere de observación y evaluación por parte de los líderes. No representa una práctica aislada de la experiencia, porque en ella se ponen en juego elementos que solo surgen como respuesta a situaciones, necesidades o intereses específicos (Van Dierendonck \& Patterson, 2015, citados en Smylie, Murphy $\&$ Louis, 2016). En este sentido, el cuidado no es un mero contrato entre dos partes, no lo mueve la coerción o la expectativa de una recompensa explícita, sino que en su base se encuentra la posibilidad de mejorar las condiciones de los demás (Lawrence \& Maitlis, 2012; Smylie, Murphy \& Louis, 2016).

- Niveles de comprensión del "caring leadership"

Un primer nivel de comprensión del caring leadership lo asociamos con las características que debe tener el líder, tanto en su práctica como en el quehacer del liderazgo. Es así que, para que se lleve a cabo la práctica del cuidado, se requiere de la mutualidad, de una relación entre dos o más actores. Cuidar a otra persona está condicionado por emociones, por afectos, por la intención de quién entrega el 
cuidado, es decir, "cuidar no es simplemente lo que la persona que cuida quiere y hace, también es la medida en que la persona cuidada percibe que esa intención y acción son afectuosas" (Smylie, Murphy $\&$ Louis, 2016, p. 6), es lo que Noddings identifica como relación "recíproca" (2001).

Esta relación es recíproca y no solo requiere de una condición de simetría, sino que puede darse entre directoras, docentes o estudiantes, incluso entre aquellos que comparten una misma posición al interior de las comunidades escolares; la mutualidad y la reciprocidad puede darse en múltiples direcciones (Smylie, Murphy \& Louis, 2016).

Desde este enfoque de liderazgo, el líder se caracteriza por ser un sujeto auténtico y empático, que atiende a los demás, se esfuerza por conocer a su equipo y genera motivación y adhesión a la escuela. Todas estas características le permiten impulsar la mutualidad y reciprocidad en las relaciones interpersonales entre los actores de la comunidad educativa, así como buscar fomentar y desarrollar las capacidades de cuidado y las relaciones sociales próximas entre todos los actores de la comunidad escolar (Smylie, Murphy \& Louis, 2016; Louis, Murphy \& Smylie, 2016; Louis \& Murphy, 2017).

Un segundo nivel de comprensión de este enfoque de caring leadership se asocia con la institucionalidad del espacio escolar, es decir, desde la posibilidad de concebir el cuidado en la conformación de objetivos y valores institucionales de las escuelas. Plasmar estas cualidades del cuidado en un documento institucional y ciertamente velar por su apropiación y cumplimiento pueden ayudar a desarrollar la capacidad de cuidado entre otros, fomentar relaciones sociales proximales, generar condiciones organizacionales de apoyo y desarrollar contextos para cuidar más allá de la escuela (Smylie, Murphy \& Louis, 2016).

En este sentido, es fundamental que las prácticas del líder sean ejemplares y coherentes con los objetivos del cuidado, por lo que sus acciones e interacciones deben ser consistentes con sus objetivos, sus virtudes y la comprensión del cuidado (Smylie, Murphy \& Louis, 
2016). De esta forma, se potenciará el desarrollo de comunidades solidarias (capacidad de cuidado entre todos), ya que, cuando los actores de la comunidad educativa se sienten atendidos y cuidados, es más probable que generen confianza en quien los cuida (Louis \& Murphy, 2017) y, por tanto, que las comunidades se transformen en espacios de colaboración, reciprocidad, trabajo colectivo, entre otras características.

Por otra parte, investigaciones de Louis, Murphy \& Smylie (2016) señalan que el caring leadership del director tiene un fuerte impacto en la responsabilidad colectiva y en el apoyo académico para el aprendizaje de los estudiantes, es decir, la mejora de este proceso está directamente relacionada con el apoyo que el líder (director) pueda entregar a sus docentes para fortalecer estas actividades. En este sentido, la mejora de los aprendizajes está asociada a construir una base de apoyo escolar amplio y positivo (Louis \& Murphy, 2018).

El caring leadership, al promover la responsabilidad colectiva, sugiere una cultura solidaria para todos los actores de la comunidad escolar, generando un sentido de responsabilidad por el bienestar de todos los integrantes de ella, porque "el cuidado de los directores también crea un clima de seguridad personal" (Smylie, Murphy \& Louis, 2016, p. 337).

\section{Metodología}

La presente investigación fue de tipo exploratorio longitudinal y con producción de conocimiento a partir de fuentes primarias. El acercamiento al objeto de estudio se realizó a partir de la metodología cualitativa, ya que ésta permite adentrase en las significaciones sociales de los individuos, sus motivaciones y las acciones sociales que ellos generan (Gaínza, 2006).

Desde esta óptica, resultó de gran importancia sumergirnos en los fenómenos desde las perspectivas y relaciones que las mismas participantes generaban en su cotidianeidad (Hernández-Sampieri y Mendoza, 2018). La relevancia estuvo puesta en el discurso de las 
directoras $^{4}$, ya que a través de ellas se reconstruyeron los sentidos sociales otorgados a su experiencia (Villegas y González, 2011).

A su vez, esta investigación fue un estudio de casos, lo que supuso un abordaje desde lo singular y lo distintivo (Stake, 1999) y permitió la profundización específica en hechos de interés, otorgando la oportunidad de captar y reflejar los elementos que dieron significado al objeto en cuestión. Así, se privilegió la idea de "profundidad" en desmedro de una gran representatividad dentro del objeto de estudio.

Para acercarnos al objeto de estudio utilizamos el método biográfico (Bolívar y Domingo, 2019), más concretamente la técnica de investigación llamada "relato de vida": expresión de una parte de la historia personal de las entrevistadas que se enlaza con un tiempo, una secuencia y un espacio específicos, configurándose así como una narración biográfica (Ibíd.). Se realizaron tres entrevistas biográficas semiestructuradas por cada caso, en el transcurso de un año, enfocadas en profundizar en diversos periodos del desarrollo profesional de las directoras que fueron relevantes para su ejercicio del liderazgo.

En esta investigación se trabajó con diez directoras de establecimientos educacionales de enseñanza básica de la Región Metropolitana de Chile. La construcción de la muestra se hizo a partir del criterio de saturación de información (Parra, 2019).

4 Con esto asumimos que esta investigación buscó acercarse a un fenómeno de las comunidades educativas desde la voz de un solo actor, por lo que el desafío sigue siendo poder entender los impactos de este fenómeno desde una perspectiva relacional y multiactoral. 
Tabla 1.

Caracterización de las directoras participantes

\begin{tabular}{|c|c|c|c|c|c|}
\hline Seudónimo & $\begin{array}{l}\text { Rango } \\
\text { Edad }\end{array}$ & $\begin{array}{l}\text { Estudios } \\
\text { formación } \\
\text { inicial }\end{array}$ & $\begin{array}{c}\text { Años de } \\
\text { experiencia } \\
\text { como } \\
\text { directora }\end{array}$ & $\begin{array}{c}\text { Año de } \\
\text { experiencia } \\
\text { en cargo de } \\
\text { dirección actual }\end{array}$ & $\begin{array}{l}\text { Dependencia } \\
\text { establecimiento }\end{array}$ \\
\hline Antonia & 55 y más & $\begin{array}{l}\text { Pedagogía } \\
\text { general básica }\end{array}$ & 2 años & 2 años & $\begin{array}{l}\text { Subvencionado } \\
\text { Particular }\end{array}$ \\
\hline Ivana & $30-39$ & $\begin{array}{l}\text { Pedagogía } \\
\text { general básica }\end{array}$ & 2 años & 2 años & $\begin{array}{l}\text { Subvencionado } \\
\text { Particular }\end{array}$ \\
\hline Macarena & $40-55$ & $\begin{array}{l}\text { Licenciada } \\
\text { en literatura/ } \\
\text { pedagogía }\end{array}$ & 2 años & 2 años & $\begin{array}{l}\text { Subvencionado } \\
\text { Particular }\end{array}$ \\
\hline Nora & $40-55$ & $\begin{array}{l}\text { Educación } \\
\text { parvularia }\end{array}$ & 2 años & 2 años & $\begin{array}{l}\text { Subvencionado } \\
\text { Particular }\end{array}$ \\
\hline Inés & 55 y más & $\begin{array}{l}\text { Pedagogía } \\
\text { general básica }\end{array}$ & 7 años & 3 años & $\begin{array}{l}\text { Subvencionado } \\
\text { Particular }\end{array}$ \\
\hline Rosario & $40-55$ & $\begin{array}{l}\text { Pedagogía } \\
\text { general básica }\end{array}$ & 10 años & 2 años & Municipal \\
\hline Vanesa & $40-55$ & $\begin{array}{l}\text { Pedagogía en } \\
\text { educación } \\
\text { diferencial }\end{array}$ & 6 años & 2 años & Municipal \\
\hline Alma & 55 y más & $\begin{array}{l}\text { Ingeniería/ } \\
\text { Pedagogía } \\
\text { general básica }\end{array}$ & 4 años & 1 años & Municipal \\
\hline Ema & 55 y más & $\begin{array}{l}\text { Estudia } \\
\text { pedagogía en } \\
\text { (Inglés) }\end{array}$ & 10 años & 3 años & Municipal \\
\hline Marlene & $40-55$ & $\begin{array}{l}\text { Pedagogía } \\
\text { enseñanza } \\
\text { media } \\
\text { (Francés) }\end{array}$ & 7 años & 2 años & Municipal \\
\hline
\end{tabular}

Fuente: Elaboración propia.

El instrumento fue validado por un grupo de cinco expertos de diversas universidades del país, todos ellos con un vasto conocimiento en temáticas de liderazgo escolar. Dicha validación estuvo centrada en asegurar la coherencia, tanto interna como externa, del guion de las entrevistas.

Para preservar el rigor metodológico, los procedimientos analíticos fueron realizados a partir del software Atlas ti ${ }^{\circledR}$ (Bolívar, 2019). Para el análisis se trabajó con la teoría fundamentada (Glaser \& Strauss, 1967; Bonilla y López, 2016). A través de ésta, se dio énfasis principalmente a los elementos comunes presentes en el conjunto de las narraciones, permitiendo abordar el fenómeno de estudio a partir 
del despliegue de una serie de fases del pensamiento inductivo. En este sentido, se trabajó desde una codificación abierta, la cual, de manera posterior, fue relacionada al conocimiento deductivo asociado con la temática estudiada.

A partir de lo anterior se generaron las siguientes categorías:

Tabla 2.

Categorías de análisis

\begin{tabular}{ll}
\hline Categoría & Breve explicación \\
\hline Promoción del cuidado & $\begin{array}{l}\text { Dice relación con el valor que dan las directoras a la práctica } \\
\text { del cuidado en el marco de su ejercicio del liderazgo, y a la } \\
\text { necesidad de fomentarla en las comunidades educativas } \\
\text { Dice relación con el valor que dan las directoras a la práctica } \\
\text { de la confianza en el marco de su ejercicio del liderazgo, y a } \\
\text { de confianza }\end{array}$ \\
$\begin{array}{l}\text { la importancia de crear espacios que fomenten su desarrollo. } \\
\text { Reciprocidad en la relación con cómo se expresa, en el discurso de las } \\
\text { confianza y el cuidado }\end{array}$ & $\begin{array}{l}\text { entrevistadas, la construcción del cuidado y la confianza } \\
\text { como practicas recíprocas }\end{array}$ \\
\hline
\end{tabular}

Fuente: Elaboración propia.

\section{Análisis de datos}

En este apartado se profundizará en los elementos comunes de los discursos de las directoras participantes del presente estudio, para ello se considerarán las categorías de análisis antes descritas (tabla 2).

\section{a) Promoción del cuidado}

Para las entrevistadas, el "cuidado" es un valor o cualidad fundamental en la caracterización de su rol como directoras, es importante en sí mismo (Noddings, 1984). Liderar desde esta arista implica concebirlo como una función social relevante en los espacios educativos, a la hora de proyectar el desarrollo de quienes participan en ellos (Louis, Murphy \& Smylie, 2016).

Lo central y lo fundamental es que la gente se sienta cuidada, que se sienta cobijada, y yo creo que eso lo he hecho (...) Solo así pueden desarrollarse por completo y podemos potenciar la participación, tanto de los profesores como de los alumnos. (Inés) 
Las directoras demuestran estar continuamente buscando desarrollar prácticas ejemplares, muchas de las cuales dicen relación con el cuidado como un elemento central. En este sentido, ocho de las directoras entrevistadas destacan la importancia de la formación continua y del aprendizaje permanente para establecer de manera efectiva sus liderazgos desde el cuidado. En esta línea, relatan la existencia de diversas instancias de formación, orientadas a potenciar el ejercicio de un liderazgo con estas características, a la vez que señalan la necesidad de seguir fortaleciendo sus competencias en este ámbito y buscando herramientas que permitan robustecer el cuidado en el marco de las comunidades educativas, cuestión fundamental para que las acciones e interacciones del líder sean consistentes con los objetivos, las virtudes y la comprensión de cuidado (Smylie, Murphy \& Louis, 2016).

[El curso] de coaching fue porque, porque en realidad yo siempre he pensado que he necesitado desarrollar otro tipo de habilidades para poder (...) para poder relacionarme bien con la gente, de manera sana, acompañarla bien en los procesos (...), pero no era para trabajar como coaching, sino que era más bien para usarlo como directora del colegio. (Marlene)

Otro elemento relevante respecto de la promoción del cuidado dice relación con que las entrevistadas señalan que esta práctica es resultado de la motivación personal, dejando en evidencia su ausencia en nivel institucional, es decir, desde la posibilidad de concebir el cuidado como un elemento importante en la conformación de objetivos y valores institucionales en las escuelas.

Desde que era profesora me preocupaba especialmente del bienestar de los niños. Después, cuando logré el cargo de dirección, quedé un poco iplop!, porque el sostenedor nunca me mencionó el cuidado de los equipos como algo que le preocupara. Se habla mucho del clima escolar, pero siempre he sentido que es más por cumplir, al final los esfuerzos por generar relaciones más sanas y espacios seguros para los profesores es algo que yo busco más por el cómo soy, no porque haya alguien pidiéndome que lo haga. (Marlene) 


\section{b) Generación de espacios de confianza}

Las entrevistadas relatan una constante búsqueda por promover la confianza en sus equipos y cultivar un sentido de justicia organizacional, contribuyendo en la promoción del bien colectivo, la participación y la inclusión (Ryan, 2016; Louis \& Murphy, 2019). Para ellas, la confianza de los equipos de trabajo es un pilar fundamental para el desarrollo de sus liderazgos, y entienden que, a partir de ésta, pueden contribuir a cambiar el clima y la cultura escolar (Louis \& Murphy, 2018).

Yo estuve dos, dos meses en este colegio solo hablando con la gente, conversando, conociéndola, y tratando de entregar confianza, como que confiaran, confiaran en mí, que... y yo confiara en ellos, y... fue un proceso súper largo de como de sanar a la gente, a los profes, a los asistentes, de hacer pequeños cambios que ellos notaran que eran como validados, y después empezar a generar como desde la participación la construcción del proyecto. (Marlene)

En esta línea, identifican que gran parte del impacto de sus prácticas depende de la posibilidad de establecer relaciones desde la confianza (Louis \& Murphy, 2018), las que, a su juicio, les ayuda en su quehacer como directoras, potenciando la participación y el compromiso colectivo de los equipos (Ryan, 2016), con los consiguientes efectos positivos en las escuelas.

Cuando llegué a este colegio los profesores participaban poco, me costaba hacer que participaran. Es impactante cómo cambia todo cuando hay confianza, cuando hay una buena relación con el equipo. Ahora todos quieren participar, porque ven más posibilidades de incidir en las decisiones que se toman. (Antonia)

La confianza, como un fenómeno multidimensional (Louis \& Murphy, 2017), permite la distribución de tareas, facilitando el trabajo y contribuyendo a la mejora del clima de la institución. En este sentido, la mayoría de las directoras se refiere a la importancia de las relaciones de confianza con sus equipos de trabajo, identificándolas como un pilar para el ejercicio de sus liderazgos. 
[Yo tengo] un equipo en el que confío y puedo, puedo delegar, ¿te fijai?, y eso es súper importante pa mi (...) porque me permite distribuir las responsabilidades y potenciar el liderazgo de los docentes. (Ivana)

Las directoras entrevistadas relatan que la construcción de confianza con sus equipos se logra a partir del trabajo cotidiano en las comunidades educativas. A su vez, estas relaciones de confianza suelen entremezclar las naturalezas descritas, ya que, si bien su cercanía tiene un componente marcadamente afectivo (Louis \& Murphy, 2017), entienden que ésta potencia el ejercicio profesional de los demás, entregando mayores herramientas para su participación y su desarrollo.

Tienes que acercarte cada día más a los docentes, preocuparte por lo que les pasa, por cómo están viviendo el día a día. Y en la medida en que van confiando en ti, su participación profesional va a ser más fructífera también fuera del aula, porque se involucran en otros espacios $y$ de otras formas. (Vanesa)

Confiar conduce a una expectativa positiva de la comunidad con la que trabajas en la escuela (Fukuyama, 1995), lo que, a la luz de los relatos de las directoras, potencia un mejor clima escolar, asegura la motivación en el trabajo y el reconocimiento de otros (De Jong \& Den Hartog, 2007), y promueve el compromiso colectivo y la participación (Ryan, 2016).

Para tener una buena gestión, tú tienes que tener un buen equipo de trabajo, y confiar en tu equipo de trabajo, pensar que las personas que trabajan ahi son los mejores, eso ayuda además a que se sientan convocados y se involucren en la toma de decisiones. (Rosario)

\section{c) La reciprocidad en el cuidado y en la confianza}

Las entrevistadas declaran que reciprocidad y confianza deben ser siempre procesos recíprocos, cuestión que implica considerar las visiones de todos los actores presentes en las comunidades educativas, lo que además contribuye al compromiso colectivo y a la construcción de una "cultura inclusiva" (León, 2012; Ryan, 2016). 
La reciprocidad es una característica fundamental del cuidado y de la confianza (Noddings, 2001; Tronto 2018), por ende, no basta solo con que el líder se preocupe de cuidar o de dar confianzas (unidireccionalmente), sino que debe también ser reconocido e impulsado por todos los actores de la comunidad escolar. En la presente investigación se trabajó solo a partir de los discursos de las directoras, cuestión que limitó profundizar en el proceso de reciprocidad y permitió observar que las directoras referencian estas relaciones de una manera unidireccional, en la que entienden que su rol debe garantizar condiciones para que los participantes de las escuelas se sientan cuidados.

Yo también tengo que brindar esa autonomía y seguridad y confianza a los que están más abajo de mí, que son los profesores, el personal de educación; entonces, como todo un hilo conductor, en la medida que uno desarrolle buenos climas laborales, confianza en los trabajadores, estimulándolos positivamente, pero también siendo muy clara cuando hay que serlo, yo creo que eso va generando condiciones para el trabajo. (Nora)

Para las entrevistadas, tanto la confianza como el cuidado forman parte de las relaciones sociales y están condicionados por emociones y afectos. En este sentido, para ellas resulta relevante entender que estos elementos no solo se refieren a la práctica de quien los ejerce, sino que también a la forma en que las demás personas los reciben y los sentimientos que generan (Noddings, 2001; Smylie, Murphy \& Louis, 2016). En esta línea, en el discurso de las entrevistadas se observa una preocupación contante por cómo son recibidas las prácticas de cuidado en las comunidades educativas.

Yo siempre estoy atenta a cómo reacciona la gente, cómo van funcionando o no los cambios que hacemos. Una busca siempre que haya respeto en el colegio y que todos convivan en espacios en los que se sientan cuidados y seguros, pero el resultado de esto solo lo podemos ver en el cómo los demás actúan frente a éste, es la única forma de saber si funciona o no. (Inés) 
Tal como señala la literatura, los docentes pueden desarrollar una visión positiva de su labor y de sus líderes cuando los superiores, como es el caso de Inés, demuestran confianza en las competencias profesionales de sus profesores (Brower, Schoorman \& Hoon, 2000).

Por esto, las directoras señalan constantemente la necesidad de generar condiciones de confianza y cuidado que permitan un vínculo recíproco con sus equipos de trabajo y la comunidad educativa. Esto les permitiría promover la seguridad, los compromisos, un mejor desempeño y el sentido de responsabilidad, elementos que actúan en pos del bienestar de todos y de la mejora escolar (Cerna, 2014; Tschannen-Moran \& Gareis, 2015; Peña, Weinstein \& Raczynski, 2018).

Yo siento que la gente, al ganar como confianza y seguridad en ellos mismos, finalmente hacen mejor el trabajo, ¿me entiendes?, como que se comprometen más, están más reflexivos... (Marlene)

Para las directoras es importante que los líderes promuevan estas cualidades, porque entienden que sus prácticas repercuten en las actividades cotidianas de los demás integrantes de las escuelas, en el entendido de que "el cuidado de los directores también crea un clima de seguridad personal” (Smylie, Murphy \& Louis, 2016, p. 337).

\section{Conclusión}

El presente artículo ha pretendido ser un aporte en relación con el rol que juegan las prácticas de confianza y cuidado en el liderazgo de directoras de establecimientos de educación básica chilena. Esta investigación fue un estudio de caso, por lo que los elementos aquí desarrollados no tienen la pretensión de ser explicativos de la problemática general estudiada, sino que más bien un primer paso exploratorio, que permita perfilar futuras discusiones en relación a cómo estos componentes resultan relevantes para el ejercicio práctico del liderazgo en el sistema educativo chileno.

Es posible señalar que existe una preocupación permanente, de parte de las directoras, por desarrollar prácticas que fomenten el 
cuidado al interior de los equipos de trabajo, y por la construcción de un clima de confianza en el marco de las relaciones que se establecen en el campo educativo.

En sus discursos es reiterado el ejercicio por fortalecer las relaciones interpersonales en sus equipos de trabajo, situación que la mayoría lo hace desde el afecto y la preocupación por el bienestar de los demás. En esta línea, por ejemplo, destaca la necesidad permanente de acercarse a los docentes y de conocer lo que les pasa y les preocupa, y el interés por formarse en habilidades que les permitan fortalecer estás prácticas; también el vínculo de confianza que construyen con los equipos de gestión, así como la búsqueda por generar espacios de seguridad en sus comunidades educativas.

A la luz de los discursos analizados, se puede relacionar las prácticas de cuidado y de construcción de confianzas, y la forma en que las directoras declaran ejercerlos, con un liderazgo escolar positivo. El cuidado y la confianza, según estas directoras, son elementos latentes que se encuentran a lo largo de todas sus experiencias de gestión, cuestión que va de la mano con la intención declarada de estar constantemente en búsqueda de la construcción de un clima escolar que permita generar condiciones favorables para el aprendizaje efectivo de los estudiantes.

Así, se observa que, para las directoras entrevistadas, tanto la construcción de confianza como el cuidado se estructuran como dos pilares para el ejercicio de sus liderazgos. En sus discursos se deja ver que son necesarios e indispensables para alcanzar la mejora en los aprendizajes, para trabajar colectiva e inclusivamente y, de esta forma, lograr fortalecer las comunidades en las que se vivencia la experiencia del aprendizaje, lo que puede conllevar una transformación de las escuelas (Louis \& Murphy, 2018).

En Chile, durante la última década, se ha puesto en valor la presencia de las relaciones intrapersonales en los instrumentos de la política educativa que guían la práctica de los líderes - El Marco para la Buena Dirección y el Liderazgo Escolar (2015) y Estándares Indicativos de Desempeño para los Establecimientos Educacionales y sus Sostenedores 
(2014)_. Pese a ello, las directoras de esta investigación señalan que su principal motivación para integrarlas en sus prácticas de liderazgo dice relación esencialmente con motivaciones personales. En esta línea, resulta relevante cuestionarnos, a futuro, cómo las instituciones educativas participan de estos procesos y el rol que juegan en ellos, sobre todo mientras la literatura internacional señala la importancia de la construcción de relaciones interpersonales sólidas a la hora de pensar la mejora y la trasformación de las comunidades educativas (Brezicha, Bergmark y Mitra, 2015; Louis \& Murphy, 2017).

A modo de cierre, resulta relevante reiterar que el presente artículo no ha pretendido generalizar ni dar respuestas concretas respecto de cómo se vivencian las prácticas de cuidado y confianza en el ejercicio del liderazgo de mujeres directoras en el contexto educativo chileno. Se entiende aquí que, para avanzar en esta línea, resulta relevante tomar en consideración la voz de todos los actores presentes en las comunidades educativas, sobre todo en el entendido de que estos componentes deben ser pensados desde una óptica relacional. La intención ha sido entregar unas primeras reflexiones acerca del rol que juegan estas prácticas en los discursos de las directoras cuando ejercen sus liderazgos.

A futuro se considera relevante seguir profundizando en esta materia y posicionándola como un tema relevante de discutir para el sistema educativo chileno. El valor de los elementos interpersonales está cada vez más presente en cómo pensar la mejora en las escuelas, por lo que profundizar en esta materia puede entregar herramientas para enfrentar los desafíos actuales de la escuela en el contexto chileno.

\section{Referencias}

Aziz dos Santos, C. (2019). La construcción de relaciones de confianza y su evaluación: Desafíos y oportunidades para el fortalecimiento de la educación pública en Chile. Líderes Educativos, Nota Técnica No 4. Valparaíso, Chile: Centro de Liderazgo para la Mejora Escolar.

Blackmore, J. (2006). Justicia social y el estudio y la práctica del liderazgo en la educación: una historia feminista. Revista de Administración e 
Historia de la Educación, 38(2), 185-200. DOI: https://doi: 10.1080 / 00220620600554876 .

Bolívar, A. (2010). El liderazgo educativo y su papel en la mejora: una revisión actual de sus posibilidades y limitaciones. Psicoperspectiva, 9(2), 9-33. DOI:10.5027/PSICOPERSPECTIVAS-VOL9-ISSUE2-FULLTEXT-112

Bolívar, A. (2012). Politicas actuales de mejora y liderazgo educativo. Málaga: Ediciones Aljibe.

Bolívar, A. (2019). Una dirección escolar con capacidad de liderazgo pedagógico. Madrid: Arco Libro-La Muralla.

Bolívar, A. y Domingo, J. (2019). La investigación (auto)biográfica en educación. Barcelona: Octaedro.

Bonilla, M. A. y López, A. (2016). Ejemplificación del proceso metodológico de la teoría fundamentada. Cinta de Moebio, 57, 305-315. DOI: http:// doi.org/10.4067/S0717-554X2016000300006.

Brezicha, K., Bergmark, U. \& Mitra, D. (2015). One Size Does Not Fit All: Differentiating Leadership to Support Teachers in School Reform. Educational Administration Quarterly, 51(1), 96-132. DOI: 10.1177 / $0013161 X 14521632$

Brower, H., Schoorman, D. \& Hoon, H. (2000). A model of relational leadership: the integration of trust and leader-member exchange. The Leadership Quarterly, 11. DOI: 10.1016 / S1048-9843 (00) 00040-0

Bryk, A. \& Schneider, B. (2002). Trust in Schools: A Core Resource for School Reform. New York: Russell Sage Foundation.

Burrello, L., Beitz, L. y Mann, J. (2016). Un manifiesto positivo: cómo las escuelas apreciativas pueden transformar la educación pública. Chicago: Elephant Rock Books.

Cavieres, E. (2014). La calidad de la educación como parte del problema. Educación escolar y desigualdad en Chile. Revista Brasileira de Educação, 19(59), 1033-1051. DOI: https://doi.org/10.1590/S141324782014000900011.

Campos, F., Valdés, R. \& Ascorra, P. (2019). ¿Líder pedagógico o gerente de escuela? evolución del rol del director de escuela en Chile. Revista Calidad en la Educación, 51, 53-84. DOI: http://dx.doi.org/10.31619/ caledu.n51.685

Cerna, L. (2014). Trust: What it is and why it matters for governance and education (Education Working Papers, 108). Paris: OCDE.

Coleman, M. (2007). Gender and educational leadership in England: A comparison of secondary headteachers' views over time. School 
Leadership and Management, 27(4), 383-399. DOI: http://doi: 383-399. 10.1080/13632430701562991.

Conejeros, M., Rojas, J. y Segure, T. (2010). Confianza: un valor necesario y ausente en la educación chilena. Perfiles Educativos, 32(129), 30-46. DOI: https://doi.org/10.22201/iisue.24486167e.2010.129.18919.

De Jong, J. \& Den Hartog, D. (2007). How Leaders Influence Employees' Innovative Behaviour. European Journal of Innovation Management, 10(1), 41-64. DOI: 10.1108 / 14601060710720546.

Dutton, J., Roberts, L. \& Bednar, J. (2010). Pathways for positive identity construction at work: Four types of positive identity and the building of social resources. Academy of management review, 35(2), 365-393. DOI: https://doi.org/10.5465/amr.35.2.zok265

Fairholm, M. \& Fairholm, G. (2000). Leadership amid the constraints of trust. Leadership \& Organization Development Journal, 21(2), 102-109. DOI: https://doi.org/10.1108/01437730010318192

Flessa, J. (2019). La investigación reciente sobre liderazgo escolar en América Latina. El valor de un enfoque propio. En J. Weinstein y G. Muñóz, Liderazgo en escuelas de alta complejidad sociocultural. Diez Miradas (pp. 317-342). Santiago: Ediciones Universidad Diego Portales .

Fukuyama, F. (1995). Trust: The social virtues and the creation of prosperity. Londres: Hamish Hamilton.

Gaínza, Á. (2006). La entrevista en profundidad individual. En M. Canales, Metodologías de la investigación social. Santiago, Chile: Lom Ediciones.

Gilligan, C. (2013). La ética del cuidado. Barcelona: Fundación Víctor Grifóls i Lucas.

Glaser, B. \& Strauss, A. (1967). The Discovery of Grounded Theory. Chicago: Aldine.

Guimaraes, D. y Valenzuela, J. (2016). Mejorando el desempeño de los estudiantes mediante el fortalecimiento del liderazgo escolar: contexto general y algunos aprendizajes en contextos de vulnerabilidad. Líderes Educativos, Nota Técnica N¹0. Chile: Centro de Liderazgo para la Mejora Escolar, Universidad de Chile.

Harris, A. \& Chapman, C. (2002). Democratic Leadership for School Improvement in Challenging Contexts. International Electronic Journal for Leadership in Learning, 6(9), 1-10. DOI: 10.7208/ chicago/9780226317755.003.0001.

Hernandez, R., Euán, R. e Hidalgo, N. (2013). Prácticas de liderazgo escolar implicado en la promoción de la justicia social: estudio de un caso en educación secundaria. Profesorado, 17(2), 263-280. 
Hernández-Sampieri, R. y Mendoza, C. (2018). Metodologías de la investigación. Las rutas cuantitativa, cualitativa y mixta. Ciudad de México: Ediciones McGraw Hill.

Horn, A. y Marfán, J. (2010). Relación entre liderazgo educativo y desempeño escolar: revisión de la investigación en Chile. Psicoperspectivas, 9(2), 82-104. DOI: 10.5027/psicoperspectivas-Vol9-Issue2-fulltext-116.

Lawrence, T. \& Maitlis, S. (2012). Care and Possibility: Enacting an Ethic of Care through Narrative Practice. Academy of Management Review, 37(4), 641-663. DOI: http://dx.doi.org/! 0.5465/amr.2010.0466.

Lee, M. \& Louis, K. (2019). Mapping a strong school culture and linking it to sustainable school improvement. Teachin and teacher education, 81, 84-96. DOI: https://doi.org/10.1016/j.tate.2019.02.001.

Leithwood, K. (2009). ¿Cómo liderar nuestras escuelas? Aporte de la investigación. Santiago: Fundación Chile.

Leithwood, K., Harris, A. \& Strauss, T. (2010). Leading school turnaround: How successful leaders (título incompleto). San Francisco: Jossey-Bass.

Leithwood, K. y Jantzi, D. (2008). Linking leadership to student learning: the contribution of leader efficacy. Educational Administration Quarterly, 44(4), 496-528. DOI: 10.1177/0013161X08321501.

Leithwood, K., Louis, K., Anderson, S. \& Wahlstrom, K. (2004). How Leadership Influences Student Learning. Nueva York, Estados Unidos: The Wallace Foundation.

León, M. J. (2012). El liderazgo para y en la escuela inclusiva. Educatio Siglo XXI, 30(1), 133-60.

Lomotey, K. \& Lowery, K. (2015). Urban schools, Black principals and Black students: The ethno-humanist identity, en M. Khalifa, N. Arnold \& C. Overton, Handbook of urban educational leadership. Lanham, Estados Unidos: Rowman and Littlefield.

Louis, K., Leithwood, K., Wahlstrom, K. \& Anderson, S. (2010). Learning from Leadership: Investigating the Links to Improved Student Learning. Nueva York, Estados Unidos: The Wallace Foundation.

Louis, K., Murphy, J. \& Smylie, M. (2016). Caring Leadership in Schools: Findings From Exploratory Analyses. Educational Administration Quarterly, 52(2), 310-348. DOI: https://doi. org/10.1177/0013161X15627678.

Louis, K. \& Murphy, J. (2017). Trust, caring and organizational learning: the leader's role. Journal of Educational Administration, 55(1), 103-126. DOI: 10.1108 / JEA-07-2016-0077. 
Louis, K. \& Murphy, J. (2018). The Potential of Positive Leadership for School Improvement. Nordic Journal of Comparative and International Education, 2(2), 165-180. DOI: https://doi.org/10.7577/njcie.2790.

Louis, K. y Murphy, J. (2019). El enfoque del liderazgo positivo y su relevancia para las relaciones en la escuela, en J. Weinstein y G. Muñóz, Liderazgo en escuelas de alta complejidad sociocultural: diez miradas (pp. 153-191). Santiago, Chile: Ediciones Universidad Diego Portales.

Luthans, F. (2002). The need for and meaning of positive organizational behavior. Journal of Organizational Behavior: The International Journal of Industrial, Occupational and Organizational Psychology and Behavior, 23(6), 695-706. DOI: https://doi.org/10.1002/job.165.

McKinsey y Company (2008). Cómo hicieron los sistemas educativos con mejor desempeño del mundo para alcanzar sus objetivos. Recuperado de http:// educacion2020.cl/documentos/informe-mckinsey-como-hicieron-lossistemas-educativos-con-mejor-desempeno-del-mundo-para-alcanzarsus-objetivos/

MINEDUC. (2014). Estándares Indicativos de Desempeño para los Establecimientos Educacionales y sus Sostenedores. Santiago, Chile: Unidad de Currículum y Evaluación.

MINEDUC. (2015). Marco para la buena dirección y liderazgo escolar. Santiago, Chile: Maval.

MINEDUC. (2019). Bases de datos del Centro de estudios. Recuperado de http:// datos.mineduc.cl/dashboards/20031/descarga-bases-de-datos-decargos-docentes/

Molinier, P. y Lagarreta, M. (2016). Subjetividad y materialidad del cuidado: Ética, trabajos y proyecto politico. Papeles del Ceic, 1, 1-14. DOI: http:// dx.doi.org/10.1387/pceic.15319.

Murphy, J. T. (2014). Creating Productive Cultures in Schools: For Students, Teachers, and Parents. Thousand Oaks: Corwin Press.

Noddings, N. (1984). Cuidar: un enfoque femenino de la ética y la educación moral. Berkeley: Prensa de la Universidad de California.

Noddings, N. (2001). Cuidado y coacción en la reforma escolar. Journal of Educational Change, 2, 35-43. DOI: https://doi. org/10.1023/A:1011514928048.

OCDE. (15 de marzo de 2018). Resultados Talis 2018. Recuperado de: https:// www.oecd.org/education/talis/TALIS2018_CN_CHL_es.pdf.

Parra, J. (2019). El arte del muestreo cualitativo y su importancia para la evaluación y la investigación de políticas públicas: Una 
aproximación realista. Opera, 25, 119-136. DOI: https://doi. org/10.18601/16578651.n25.07.

Peña, J., Weinstein, J. y Raczynski, D. (2018). Construcción y pérdida de la confianza de docentes: un análisis de incidentes críticos. Psicoperspectiva, 17(1), 1-13. DOI: http://dx.doi.org/10.5027/ psicoperspectivas-vol17-issuel-fulltext-1174.

Riley, K. (2013). Walking the leadership tightrope: building community cohesiveness and social capital in schools in highly disadvantaged urban communities. British Educational Research Journal, 39(2), 266286. DOI: https://doi.org/10.1080/01411926.2012.658021

Robinson, V., Hohepa, M. \& Lloyd, C. (2009). School leadership and student outcomes: Identifying what works and why: best evidence synthesis iteration. Wellington, Nueva Zelanda: New Zealand Ministry of Education.

Ryan, J. (2016). Un liderazgo inclusivo para las escuelas, en J. Weinstein (Ed.), Liderazgo educativo en la escuela. Nueve miradas (pp. 77-204). Santiago, Chile: Centro de Desarrollo de Liderazgo Educativo (CEDLE) y Ediciones Universidad Diego Portales.

Shaked, H. \& Schechter, C. (2017). Systems Thinking for School Leaders: Holistic Leadership for Excellence in Education. Cham, Switzerland: Springer. Leadership and Policy in Schools, 17(4), 647-649. DOI: 10.1080/15700763.2017.1398339.

Seligman, M., Randal, E., Gillham, J., Reivich, K. \& Linkins, M. (2009). Positive education: Positive psychology and classroom interventions. Oxford review of education, 35(3), 293-311. DOI: $10.1080 / 03054980902934563$.

Smylie, M., Murphy, J. \& Louis, K. (2016). Caring School Leadership: A Multi-Disciplinary, Cross-Occupational Model. American Journal of Education, 123(1), 1-35. DOI: 10.1086 / 688166.

Smylie, M, Mayrowetz, D., Murphy, J. \& Louis, K. (2007). Trust and the development of distributed leadership. Journal of School Leadership, 17(4), 469-503. DOI: 10.1177/105268460701700405.

Stake, R. (1999). Investigación con Estudio de Casos. Madrid, España: Ediciones Morata.

Torre, D. \& Murphy, J. (2014) Communities of parental engagement: new foundations for school leaders' work. International Journal of Leadership in Education, 19(2), 203-223. DOI: 10.1080/13603124.2014.958200.

Tronto, J. (2018). Economía, ética y democracia: tres lenguajes en torno al cuidado, en L. Arango, Género y cuidado. Teorias, escenarios y politicas (pp. 22-37). Bogotá: Publicación Universidad Nacional de Colombia. 
Tschannen-Moran, M. (2009). Fostering teacher professionalism in schools: the role of leadership orientation and trust. Educational Administration Quarterly, 45(2), 217-247. DOI: https://doi. org/10.1177/0013161X08330501

Tschannen-Moran, M. \& Gareis, C. (2015). Principals, trust, and cultivating vibrant schools. Societies, 5(2), 256-276. DOI: 10.1007 / 978-3-31950980-8_8.

Umekubo, L. A., Chrispeels, J. y Daly, A. (2013). Estrechos lazos en un distrito descentralizado: estudio de caso de un distrito que mejora. Pensamiento Educativo, 50(2), 69-96. DOI: 10.7764/PEL.50.2.2013.5

Vanni, X., Bustos, N., Valenzuela, J. \& Bellei, C. (2018). The role of leadership in low-performing schools: The Chilean case, en C. Meyers \& M. Darwin, School turnaround and reform: Vol. 2. International perspectives on leading low-performing schools. Charlotte: IAP.

Villegas, M. y González, F. (2011). La investigación cualitativa de la vida cotidiana. Psicoperspectivas, 10(2), 35-59.

Wahlstrom, K. \& Louis, K. (2008). How teachers experience principal leadership: the role of professional community, trust, efficacy and distributed responsibility. Educational Administration Quarterly, 44(4), 458-495. DOI: 10.1177/0013161X08321502.

Waters, T., Marzano, R. \& McNulty, B. (2003). Balanced Leadership: What 30 Years of Research Tells Us about the Effect of Leadership on Student Achievement. Aurora, CO: Mid-Continent Research for Education and Learning.

Weinstein, J. y Muñóz, G. (2012). ¿Qué sabemos sobre los directores de escuela en Chile? Santiago: CEPPE-Fundación Chile.

Weinstein, J., Raczynski, D. \& Peña, J. (2018). Relational trust and positional power between school principals and. Teachers in Chile: A study of primary schools, 48(1), 64-81. DOI: https://doi. org/10.1177/1741143218792912.

Youngblade, L., Theokas, C., Schulenber, J. \& Curry, L. (2007). Risk and promotive factors in families, schools, and communities: A contextual model of positive youth development in adolescence. Pediatrics, 119(1), 47-53. DOI: 10.1542 / peds.2006-2089H.

Recibido: 01/04/2020

Aceptado: 12/11/2020 